\title{
Kaposi's sarcoma-associated herpesvirus ORF57 promotes escape of viral and human IL6 RNAs from microRNA-mediated suppression
}

\author{
Jeong-Gu Kang ${ }^{1 *}$, Natalia Pripuzova', Vladimir Majerciak', Shu-Yun Le², Zhi-Ming Zheng ${ }^{1}$ \\ From $12^{\text {th }}$ International Conference on Malignancies in AIDS and Other Acquired Immunodeficiencies \\ (ICMAOI) \\ Bethesda, MD, USA. 26-27 April, 2010
}

Cancerous cells transformed by KSHV infection manifest an increased expression of both viral and human interleukin 6 (IL6). Although increased IL6 appears important to maintain cancer cell proliferation, what causes IL6 increase in the tumor cells by KSHV infection remains largely unknown. KSHV mRNA transcript accumulation protein (MTA or ORF57) has been characterized as a multifunctional protein in regulation of viral gene expression.

In seeking for genome-wide RNA targets of MTA by the CLIP assay, we identified viral IL6 (vIL6) as an MTA target which contains an MTA response element (MRE). MTA enhanced both vIL6 and human IL6 (hIL6) expression and cells with a MTA-null KSHV genome displayed a deficiency in vIL6 expression during virus lytic induction. Mutations in the vIL6 MRE identified its role in translational enhancement of vIL6, suggesting that vIL6 is translationally regulated in a sequence-specific manner. Unexpectedly, Ago2 which is a major component in RISC was found in RNA-protein pulldown assays by using MRE-RNA oligomers. Bioinformatics analysis showed that the identified vIL6 MRE contains a functional seed match to miR-1293. Ectopic expression of miR-1293 prevented vIL6 expression. Mutation of the miR-1293 seed match in the MRE or by ectopic expression of MTA could diminish the translational repression of vIL6 mediated by miR-1293 in vitro and in vivo, resulting in enhancement of vIL6 expression. Furthermore, we demonstrated that hIL6 expression is also under control by miR-608 binding to a similar MRE in the corresponding region. Through interaction with a RISC component Ago2, MTA prevents miRNA-mediated recruitment of IL6 mRNA into RISC, thereby relieving the translational repression.

Together, our results suggest the existence of a highly conserved miRNA pathway in cells in prevention of cytokine-induced cell proliferation and compelling evidence of how an oncogenic virus invades this pathway to induce cell proliferation and tumorigenesis during virus infection.

\section{Acknowledgements}

This article has been published as part of Infectious Agents and Cancer Volume 5 Supplement 1, 2010: Proceedings of the $12^{\text {th }}$ International Conference on Malignancies in AIDS and Other Acquired

Immunodeficiencies (ICMAOI). The full contents of the supplement are available online at http://www.biomedcentral.com/1750-9378/5?issue=S1.

\section{Author details}

'HIV and AIDS Malignancy Branch, CCR, NCl, Bethesda, MD, USA.

${ }^{2}$ Nanobiology Program, CCR, NCl, Bethesda, MD, USA.

Published: 11 October 2010

doi:10.1186/1750-9378-5-S1-A45

Cite this article as: Kang et al:: Kaposi's sarcoma-associated herpesvirus ORF57 promotes escape of viral and human IL6 RNAs from microRNAmediated suppression. Infectious Agents and Cancer 2010 5(Suppl 1):A45. 